\section{Hypocalcaemia in a patient with congenital heart disease}

\section{A McCusker N P Jenkins J E Hancock}

J R Soc Med 2007; 100:51-53

The 22q11 microdeletion syndrome should be considered in both paediatric and adult patients when hypocalcaemia occurs in the presence of congenital heart disease, facial dysmorphism or learning difficulties.

\section{CASE REPORT}

A 32-year-old woman with learning difficulties presented with malaise and acute abdominal pain. She had had a cleft palate and an atrial septal defect repaired in childhood, and was known to have a persisting ventricular septal defect. Computed tomography revealed a splenic infarct. Blood cultures subsequently grew streptococcus anginosus, and an echocardiogram demonstrated a large vegetation attached to the tricuspid valve (Figure 1). A diagnosis of bacterial endocarditis was established and she was treated successfully with a prolonged course of antibiotic therapy. Her illness was relatively protracted, and was complicated by pneumonia, but she eventually made a full recovery.

During her admission, she was noted incidentally to have profound hypocalcaemia (corrected serum calcium $1.79 \mathrm{mmol} / \mathrm{L})$, though this was not associated with any major clinical signs or symptoms. The alkaline phosphatase was within the normal range, and there was relative parathyroid hormone deficiency $(58 \mathrm{pg} / \mathrm{ml})$. In view of her past history and facial appearance (Figure 2a and b), diGeorge syndrome was considered as a potential unifying diagnosis, and this was subsequently confirmed by demonstration of a 22q11 microdeletion using in situ hybridization with the Vysis TUPLE1 probe (Figure 3). She was treated with calcium and vitamin D supplementation and her serum calcium returned to normal. Her eight-yearold son, who had had mild behavioural problems, was also screened and was found to have an identical 22q11 microdeletion. Although her parents were not screened, there was no suggestive history of the condition, and this was assumed to have started as a new mutation.

Department of Cardiology, Wythenshawe Hospital, Southmoor Road, Wythenshawe M23 9LT, UK

Correspondence to: Dr L McCusker, 8 Robert Harrison Avenue, Manchester M20 1LW, UK

E-mail: louisemccusker03@hotmail.com

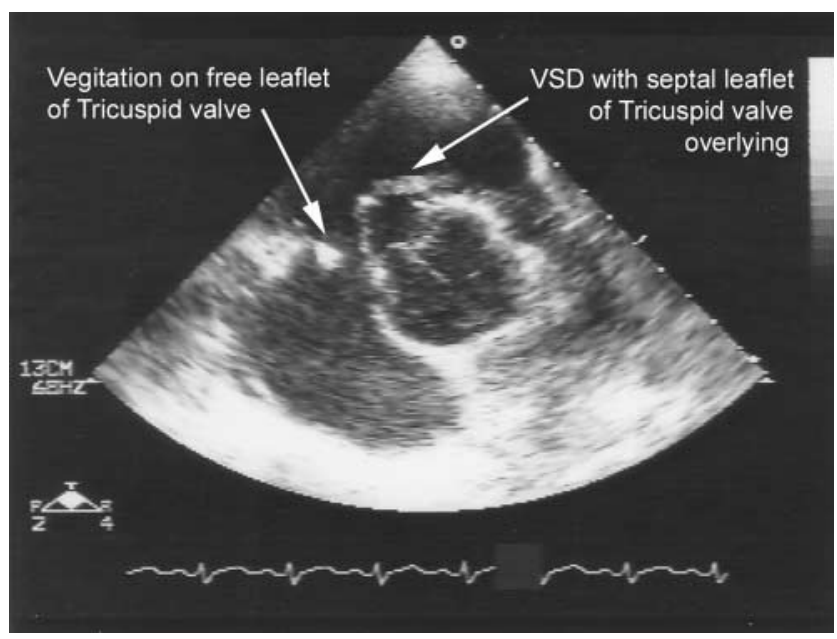

Figure 1 Echocardiogram with short axis parasternal view demonstrating tricuspid valve endocarditis

\section{DISCUSSION}

diGeorge syndrome, and the related disorders Shprintzen syndrome (velocardiofacial) and Takao syndrome (conotruncal anomaly face), arise because of a microdeletion at the locus 22q11.2, resulting in abnormal branchial arch development during embryogenesis., ${ }^{1,2}$ These clinical syndromes have previously been considered under the general term 'CATCH-22', an acronym incorporating the major common overlapping characteristics (Cardiac defects, Abnormal facies, Thymic hypoplasia/aplasia, $\underline{\text { Cleft palate, }}$ Hypocalcaemia, deletion 22q11), ${ }^{1,3}$ but are now more usually referred to as the '22q11 microdeletion syndrome' to reflect their shared pathology.

Historically, the incidence of the 22q11 microdeletion was thought to be in the region of 1 in 20,000 live births, ${ }^{3}$ but contemporary reports have suggested that the incidence may be closer to 1 in 4000, making it amongst the most common human chromosomal abnormalities. ${ }^{4}$ This apparent rise in incidence probably reflects increasing clinical awareness and improved genetic techniques for detection, rather than a genuine epidemiological shift. There appears to be no gender or ethnic predilection.

The 22q11 microdeletion is thought to result in abnormal migration of neural crest cells at a critical point in organogenesis, resulting in abnormal development of those structures arising from the branchial arch system, including the face, thymus gland, parathyroid gland, aorta, and major vessels of the head and neck. ${ }^{4}$ More than eighty different malformations and birth defects have been described in association with the 22q11 microdeletion, thus giving rise to a large number of diverse but overlapping clinical syndromes. ${ }^{4}$ The condition is usually diagnosed shortly after birth due to the presence of facial dysmorphism or cardiac abnormalities, and the clinical 
(a)

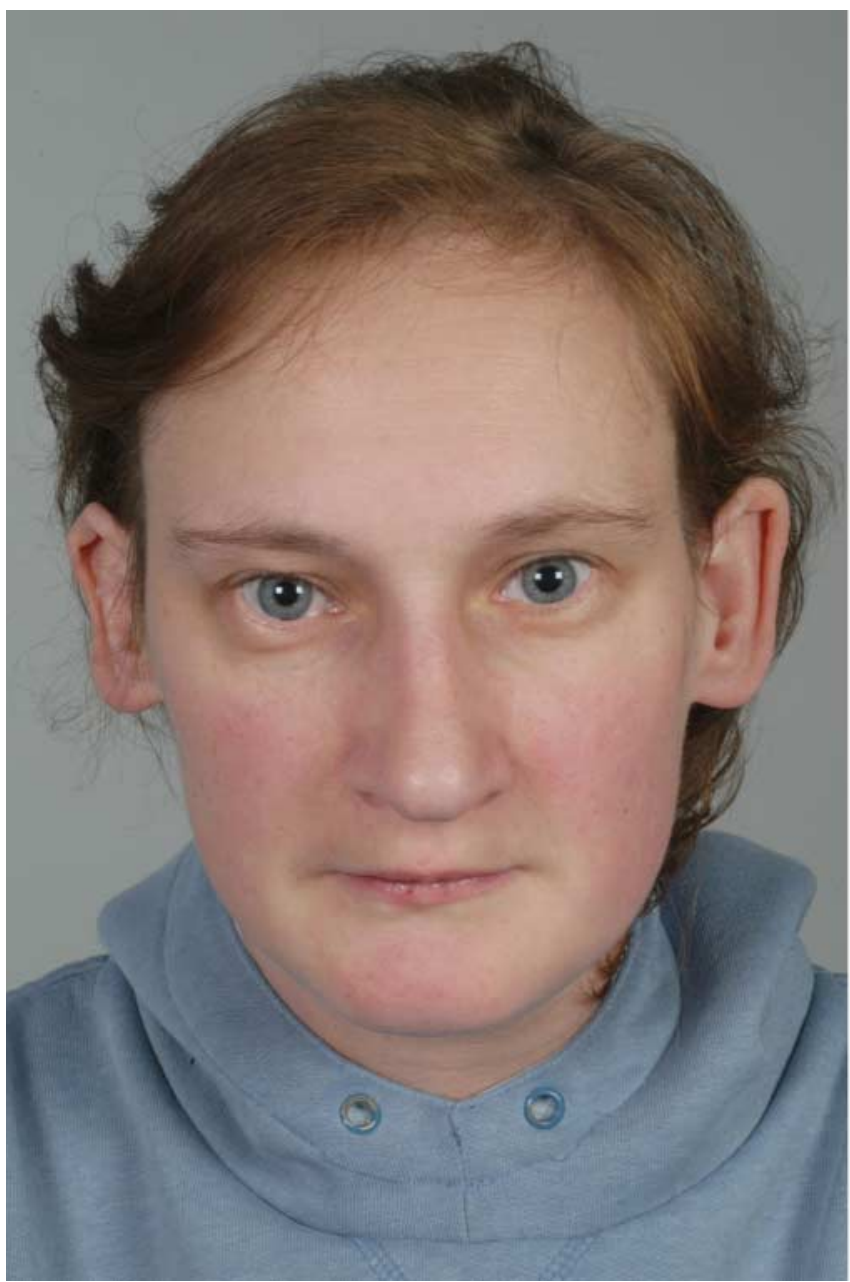

(b)

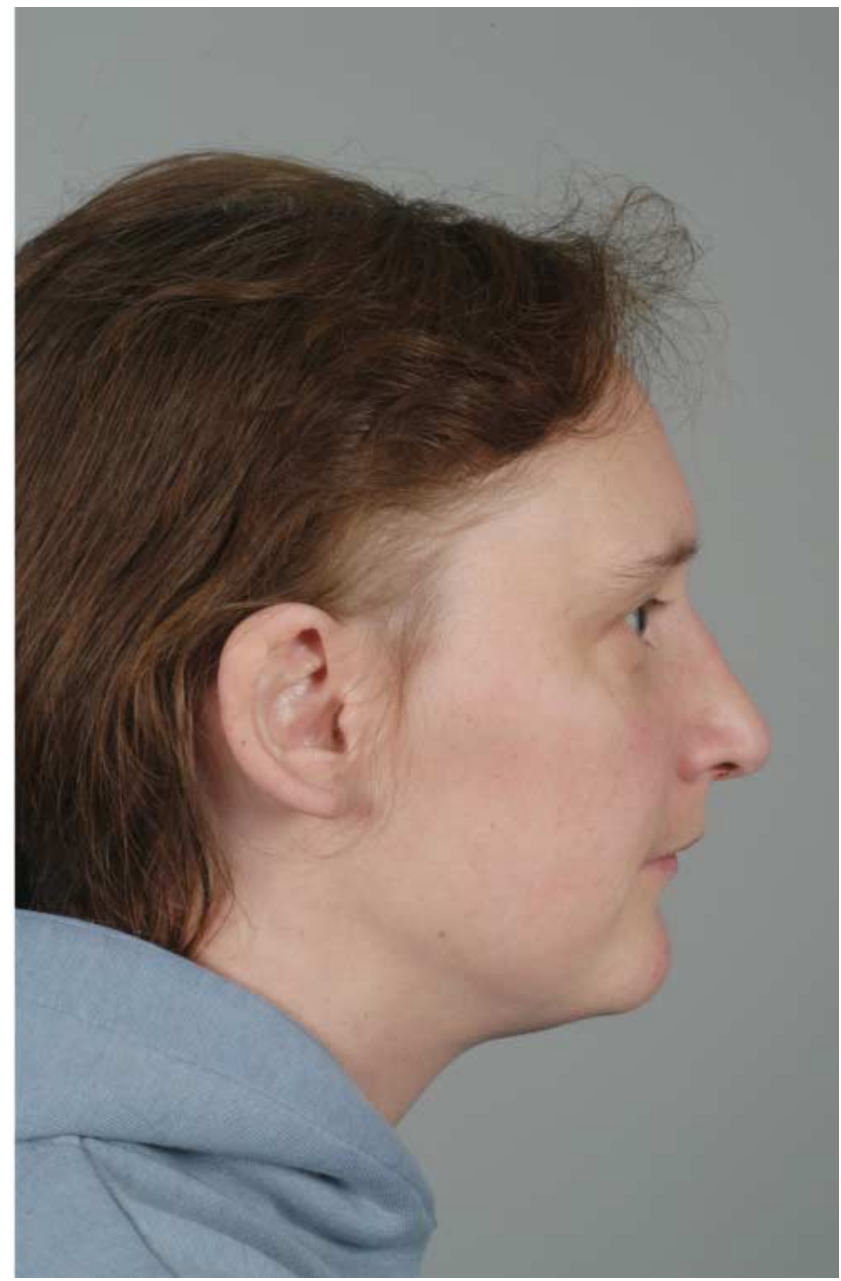

Figure 2 (a) Frontal view of patient illustrating telecanthus; (b) Lateral view illustrating short philtrum, microretrognathia and low set ears. In colour online

features may be confused with those of the foetal alcohol syndrome. ${ }^{3}$ Patients with milder clinical features may go undetected until adulthood, and some patients may remain undiagnosed throughout life. In part, this often reflects the involvement of multiple unrelated subspecialties throughout different stages of child and adulthood, which may mitigate against the establishment of a unifying diagnosis.

Patients with the 22q11 microdeletion have distinctive facies with low set ears, occasionally defective pinnae, microretrognathia (a small recessed mandible), hyperelorism (abnormally widely spaced orbits) and a short philtrum (distance between the upper lip and nares). They may have a relatively small mouth and telecanthus (increased distance between inner canthi) with short palpebral fissures (eye slits). Cleft palate, lip or uvula may also be present. The severity of facial dysmorphism is
Cardiac abnormalities are common and occur in approximately $75 \%$ of all patients, typically consisting of conotruncal defects, mainly affecting the base of the heart and origin of the great vessels, including truncus arteriosus, tetralogy of fallot, interrupted aortic arch, and pulmonary atresia, though ventricular septal defects also occur relatively frequently. ${ }^{5}$ Congenital heart defects have been identified as the main cause for morbidity and mortality in patients with the 22q11 microdeletion. ${ }^{4}$

Thymus gland development may be affected in some individuals leading to thymic hypoplasia or aplasia, and subsequently to impaired T-cell immunity, which may manifest as repeated respiratory infections. Often, however, immunodeficiency is relatively mild and may not be suspected clinically. ${ }^{3}$ diGeorge syndrome has also been associated with autoimmune diseases including Grave's disease, and patients remain at increased risk of graft versus 


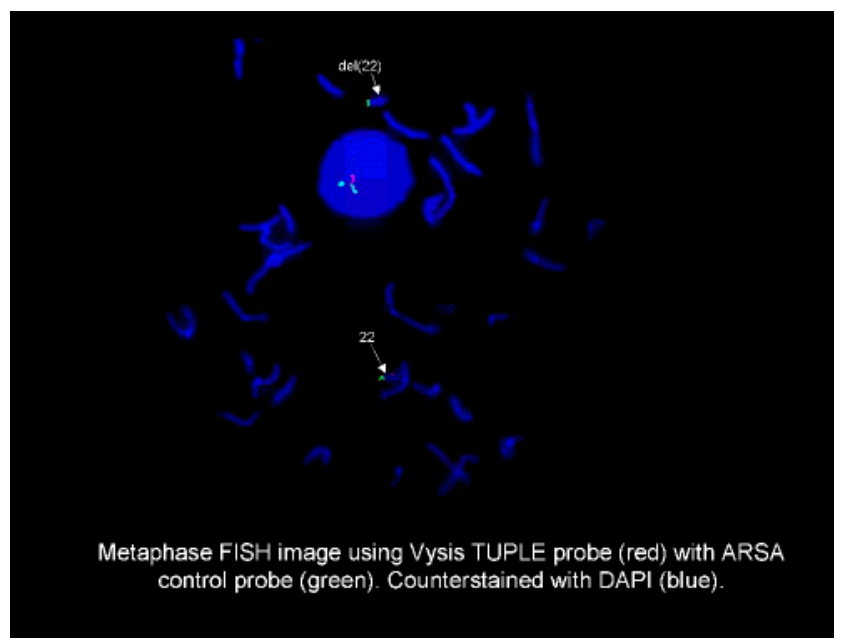

Figure 3 Diagnosis by Fluorescence In situ Hybridization (FISH). In colour online

host reactions during blood transfusion. ${ }^{3}$ Hypocalcaemia due to maldevelopment of the parathyroid glands is also relatively common and may present with seizures or tetany during childhood.

Neuro-psychiatric abnormalities also commonly accompany the physical manifestations associated with the 22q11 microdeletion. Learning difficulties and behavioural problems occur frequently in childhood, and although there appears to be wide individual variation, several studies have reported a mean IQ of approximately 75, with many patients having specific deficits of language and speech development. ${ }^{4}$ Some studies have also reported an increased incidence of psychotic illness, including schizophrenia and bipolar disorder subsequently in adulthood. ${ }^{4}$

In the majority of cases, the 22q11 microdeletion arises as a de novo mutation, but in approximately $8 \%$ of cases the condition is familial with autosomal dominant inheritance.
Although there is much debate about the benefits of prenatal diagnosis, fluorescence in situ hybridization (FISH) testing should be offered to those families who have had a child previously affected by the mutation or if a parent is affected by the condition and has the 22q11 microdeletion. ${ }^{6}$ Prenatal diagnosis may also be helpful when a conotruncal cardiac defect is discovered in utero, as in this setting the prevalence of the 22q11 microdeletion is up to $48 \% .{ }^{6}$

The case report illustrates how the 22q11 microdeletion syndrome may remain undiagnosed for many years even in adult patients. The patient had many features of the condition, including learning difficulties, congenital heart disease, a previous history of cleft palate and hypocalcaemia. Timely and accurate diagnosis depends upon an awareness of these disparate features, coupled with specific genetic testing using in situ hybridization techniques. Knowledge of the presence of the 22q11 microdeletion may alert physicians to other potential manifestations and is relevant to prenatal counselling and genetic testing.

Competing interests None declared.

\section{REFERENCES}

1 Hong R. The Di George anomaly (CATCH 22, DiGeorge/ velocardiofacial syndrome). Semin Hematol 1998;35:282-90

2 Markert ML, Boeck A, Hale LP, et al. Transplantation of thymus tissue in complete DiGeorge syndrome. New Engl J Med 1999;341:1180-9

3 Sediva A. Bartunkova J. Zachova R, et al. Early development of immunity in DiGeorge syndrome. Med Sci Monit 2005;11:CR182-7

4 Scrambler PJ. The 22q11 deletion syndromes. Human Mol Genet 2000;9:2421-6

5 Goldmuntz E, Emanuel B. Genetic disorders of cardiac morphogenesis. Circ Res 1997;80:437-43

6 Driscoll DA. Prenatal diagnosis of the 22q11.2 deletion syndrome. Genet Med 2001;3:14-8 